\title{
Acupuncture meridians and their internal dependence reflected in the ancient laws for practical use
}

\begin{abstract}
The article reveals the ancient laws of acupuncture, which reflect the functional dependences of the organs and their corresponding meridians. Every acupuncturist should consider them when doing acupuncture treatments. The mother-son law reflects the strong functional dependence among the organs, while husband-wife law reflects the weak dependence among them. Some laws reflect the daily rhythms of organs' activity, while others reflect their monthly and yearly rhythms.
\end{abstract}

Volume II Issue 3 - 2018

\section{Maria Kuman}

Holistic Research Institute, USA

Correspondence: Maria Kuman, Holistic Research Institute, I4I 4 Barcelona Dr., Knoxville, TN 37923, USA,

Email holisticare@mariakuman.com

Received: May 03, 2018 | Published: June 12, 2018

\section{Introduction}

Every organ is represented on the skin surface by a specific acupuncture meridian. In the ancient acupuncture books there are two types of organs: hollow (Yang) and solid (Yin). ${ }^{1}$

Hollow Organs are the stomach, bladder, gall bladder, and the small and large intestines. Being hollow they contract more intensely or they are more active. Since 'active' means 'Yang', the Hollow Organs are Yang organs. They are represented on the surface of the skin by Yang meridians, which run downward along the back of the body and the outer side of arms and legs. The Governing (GV) meridian runs downward at the center of the back and head. (See the book of the author: M. Kuman, Modern Aspects of Ancient Acupuncture). ${ }^{3}$

Solid Organs are the liver, spleen, lungs, kidneys, and the heart. Being solid, they do not contract as actively as the hollow organs. Since 'less active' or 'passive' means 'Yin', the Solid Organs are Yin Organs. They are represented on the surface of the skin by Yin meridians, which run upward along the front of the body and on the inner side of arms and legs. The Meridian of Conception (CV) runs upward at the center of the abdomen and chest $^{2}$ (See also the book of the author: M. Kuman, Modern Aspects of Ancient Acupuncture). ${ }^{3}$

Acupuncture aims to restore the balance and normal function of our internal organs through treatments of their representing acupuncture meridians on the skin surface (see the author's book: Maria Kuman, Modern Aspects of Ancient Acupuncture). ${ }^{3}$

\section{The law of five elements in the ancient acupuncture} theory

In the ancient acupuncture book, ${ }^{1,2}$ the organs are divided into five couples of organs. In my book, ${ }^{3}$ it is explained that the organs forming a couple are always functionally closely related and they are represented on the body surface by adjacent meridians. These meridians form closed circles because they consists of one of the Yang meridians running downward on the outer side of arms, legs, and back, and one of the Yin meridians running upward on the inner side of arms, legs, abdomen and chest.
Coupled organs are:

a. Liver-Gallbladder (LV-GB)

b. Spleen-Stomach (SP-ST)

c. Heart-Small Intestines (HT-SI)

d. Lungs-Large Intestines (LU-LI)

e. Kidneys-Bladder (KI-BL).

In the ancient acupuncture book, the body energy Chi circulates in the following way:

i. First, the Liver and Gallbladder are most active.

ii. Second, the Spleen and the Stomach are most active.

iii. Third, the Heart and the Small Intestines are most active.

iv. Fourth, the Lungs and Large Intestines are most active.

v. Fifth, the Kidneys and the Bladder are most active.

In other words the energy Chi circulates following the arrows along the pentagram in Figure 1. Most of the contemporary acupuncture books have this pentagram reflecting the circulation of energy among the five couples of organs.

In some ancient acupuncture books, six couples of organs are listed and the elements of Heaven and Earth correspond to the sixth couple. In some books ${ }^{2}$ the octahedral form on Figure 2 was chosen to represents the six couples of organs, which includes the Earth below and the Heaven above (see the book Chasing the Dragon's Tail). ${ }^{2}$

Since the circulation of energy in the hollow (Yang) organs and the solid (Yin) organs is quasi-independent, in the book of the author Maria Kuman, Modern Aspects of Ancient Acupuncture ${ }^{3}$ the solid and hollow organs were separated. This transformed the representing figure of the octahedral on Figure 2 to the Tetragrammaton on Figure 3 , where the pyramid with top up represents the more active (Yang) hollow organs and the pyramid with top down - the solid less active (Yin) organs. 
Hebrew ancient texts explain that the God-creator Yahweh has the geometric shape of Tetragrammaton (see Maria Kuman, Science Speaks to God). ${ }^{4}$ Since God created everything in His image, the geometric shape of all his creations, including the human electromagnetic field, must have the symmetry of Tetragrammaton. Indeed, Sun, Earth, and Man have the same type of donut-shaped electromagnetic field (Tetragrammaton), which is the field of all selfsustained self-organized systems. ${ }^{4}$

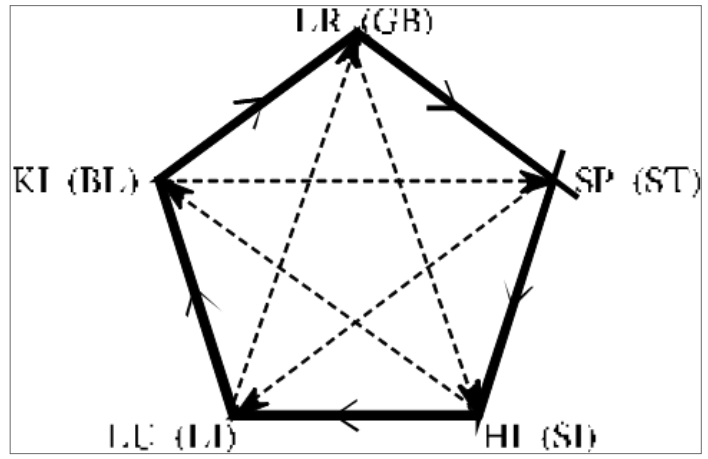

Figure I The energy Chi circulates following the arrows along the pentagram.

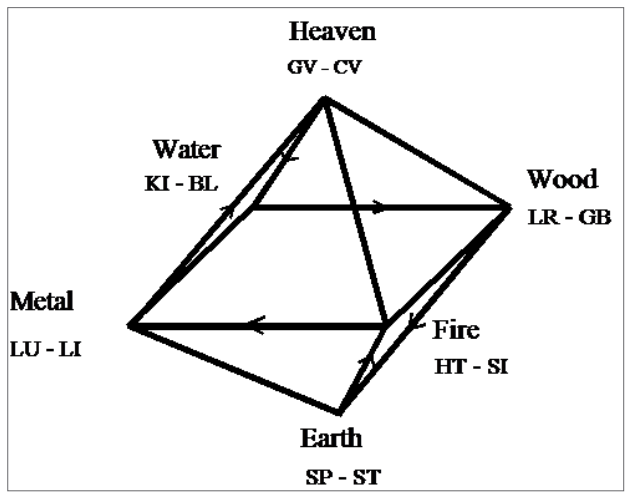

Figure 2 the octahedral form was chosen to represents the six couples of organs, which includes the Earth below and the Heaven above.

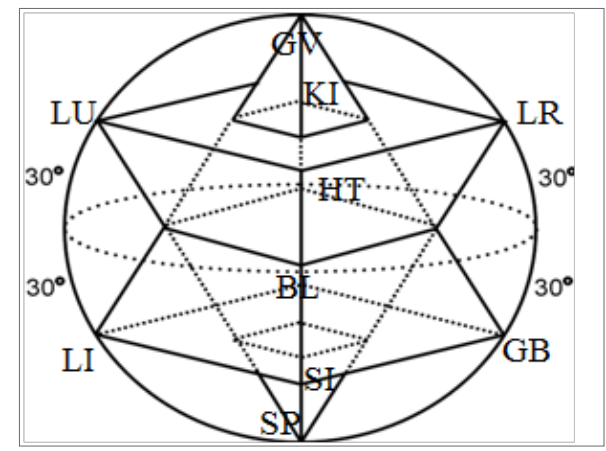

Figure 3 Tetragrammaton.

\section{Daily energy circulation in the solid and hollow organs}

According to the ancient book Huang Ti Nei Ching Sue Wen, which Ilza Veith translated in English in 1972, ${ }^{1}$ the daily circulation of energy among the solid (Yin) organs is the following:

a. From midnight to dawn the liver is most active.

b. From dawn to noon the heart is most active. c. From noon to evening the lungs are most active.

d. From evening to midnight the kidneys are most active.

It is also called midday-midnight law. Every acupuncturist must consider the midday-midnight law of organ activity when treating patients because it has been confirmed by contemporary science.

\section{From midnight to dawn the liver is most active}

Contemporary study of Dr. Halberg ${ }^{6}$ showed that the liver is maximally active after midnight. Therefore, the ancients are right that "from midnight to dawn the liver is most active". The liver is the body's main laboratory, which actively regenerates cells with limited life span, such as: skin, nails, blood cells, etc. Since regeneration means synthesis of new cells and cells' division requires water, after midnight the liver cells are three times larger than they are at noon because they contain three times more water.

\section{From dawn to noon the spleen (and stomach) are most active}

The spleen and stomach are coupled organs because their functions are closely related. According to the ancient acupuncture book, ${ }^{1}$ they are maximally active in the morning between dawn and noon. Contemporary study confirmed this. Dr. Feingin \& coworkers ${ }^{7}$ were the first to find and publish that the stomach is maximally active in the morning. ${ }^{5}$

They demonstrated that the consumption of one half kilogram of liver in the morning at $8 \mathrm{a} . \mathrm{m}$. led to a very swift rise of amino acid level in the blood, while the evening consumption at 8 p.m. of the same amount did not show any assimilation; the blood level of amino acids remained at the fasting value. ${ }^{5}$

Therefore, the ancient Hindu wisdom: Eat your breakfast alone, share your lunch with a friend, and give your supper to your enemies, seems to be right. This is the healthy way of eating; the breakfast should be the biggest meal of the day because the assimilation of proteins is maximal in the morning.

\section{At noon the heart is most active}

Contemporary study of Dr. Spoor and Dr. Jackson from the University of South Dakota found that the effect of acetylcholine on heart contraction depended strongly on the time the heart was taken out of the body. ${ }^{8}$ The effect was strongest when the heart has been taken out of the body at 11a.m., which is at the beginning of the heart's active period. The effect was weakest when the heart was taken out of the body at 11p.m. (12hours later), when the heart activity is minimum. Therefore, the contemporary study did confirm that the heart is most active at noon.

\section{From noon to evening the lungs are most active}

Contemporary study of Dr. Klein and coworkers confirmed this ancient wisdom. They found that pilots at 3a.m. use twice less oxygen than the amount they need in 3p.m. They reported their findings in the magazine Aerospace Medicine. ${ }^{9}$ Since the oxygen consumption was twice more at 3p.m. than at 3a.m., this confirms the claim of the ancients that from noon to evening the lungs are most active.

\section{From evening to midnight the kidneys are most active}

Contemporary study of Dr. F. Halberg and coworkers showed that the kidneys are maximally active at $8 \mathrm{p} . \mathrm{m} .{ }^{5,10}$ This confirms the claim 
of the ancients that from evening to midnight the kidneys are most active.

Kidneys and the bladder are coupled organs because they are functionally closely related. At regular consumption of food and water, the excretion of liquid was found to be maximal between evening and midnight and minimal between midnight and dawn. ${ }^{5}$ After midnight, urination is much less because the water is kept in the body and used by the liver for regeneration of cells.

Thus, ancient and contemporary sources give the same time of organ's activity: starting from midnight the most active are liver and gallbladder, then stomach and spleen, then heart and small intestines, then lungs and large intestines, then kidneys and bladder, then again the liver, etc. In the ancient acupuncture teaching, this is the pathway, which the energy (Chi) follows.

\section{“O”-ring and lotus posture}

Both ancient acupuncture books ${ }^{1}$ and Yoga books (M. Kuman, Yoga-Health Benefits, Science, and Wisdom $)^{11}$ claim that the energy cycle starts with breathing, which plays a leading role in the body energy cycle, and finishes with the colon or large intestines, which relate to elimination.

The lung meridian ends at the tip of the thumb, where the last acupuncture point LU 11 is. The meridian of large intestines (LI) starts at the end of pointing finger. For that reason, when you touch the tips of the thumb and the pointing finger, you close the body energy cycle. This is called "O"-ring. ${ }^{3,11}$

Since ancient times the "O"-ring has been used to test herbs, drugs, or food to find out if they would increase the body energy or not. Place the tested substance on your palm and try to disconnect the ' $O$ '-ring by pulling the thumb with the pointing finger of the other hand.

a. If it is more difficult to disconnect the ring when the substance is on the palm, the substance will increase the body energy.

b. If it is easier to disconnect the ring, the substance will decrease the body energy.

Since ancient times, Yogi do meditation in "Lotus" posture sitting on the ground with legs folded. The arms rest on the knees with palms up and the thumb and pointing fingers connected in "O-ring", which closes the energy cycle of the body. During meditation all the sensors are shut down to eliminate the external world, the conscious mind is switched off, and the body energy cycle is closed through the "O-ring". (For more details see the author's book: Maria Kuman, Yoga - Health Benefits, Science, and Wisdom). ${ }^{11}$

\section{Yearly energy circulation in the solid and hollow organs}

In Figure 4, the organs are plotted in the outer ring. In the next ring, Roman numbers indicate the month of maximal activity of each organ. In the ring after it, Arabic numbers indicate the hour of the organs' daily maximal activity. For example, the liver (LR) is maximally active during the months of January - February and in hour 23 , which is $11 \mathrm{p} . \mathrm{m}$.; the Lungs (LU) are maximally active during the months of September - October and in hour 15, which is 3p.m., and so forth.

In the ancient acupuncture book, ${ }^{1}$ during the year the organs become maximally active in the same sequence as in the daily cycle:
Liver $->$ Heart $->$ Spleen $->$ Lungs $->$ Kidneys $->$ etc., but the cycle is completed for one year instead of one day:

a. The Liver is most active in the spring.

b. The Heart $(\mathrm{PC}=$ pericardium $)$ is most active in the summer.

c. The Spleen is most active in the late summer.

d. The Lungs are most active in the fall.

e. The Kidneys are most active during the winter.

Are there contemporary studies indicating that this is true?

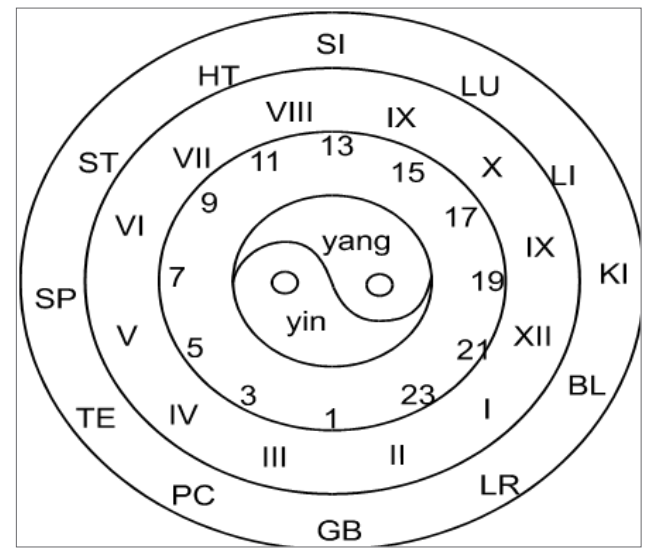

Figure 4 Yearly Energy Circulation in the Solid and Hollow Organs.

\section{The liver is most active in the spring}

Every experienced practitioner of internal medicine knows that the liver and gallbladder attacks are most frequent in the spring, which means that the coupled organs liver-gallbladder are maximally active in the spring.

In winter time the activity is low-the body temperature is low and the metabolism is slow. As a result toxins accumulate in the way dirt accumulates at places where the river is slow. In the spring as the temperature outside increases, the body metabolism speeds up. Since liver detoxifies the body, the liver is the organ that must deal with the winter toxins, which makes the liver most active during spring.

The habit of fasting in early spring did not originate in Christianity; it was incorporated in it. Fasting in early spring exists also in Muslim and other religions. The purpose of spring fasting is to let the body burn the winter toxins.

The habit of eating many eggs during Easter in later spring is as old as the world; it did not originate in Christianity, it was incorporated in it. Since the digestion of eggs require secretion of bile juice by the gall-bladder, eating of many eggs during Easter serves the purpose of cleansing the gallbladder from the winter leftover impurities, which prepares the body for the higher metabolic rates coming with the higher temperatures in the spring.

Using statistical data of the Department of Health in Minnesota, Dr. Halberg found that the suicides are maximal in May. This is because the spring is the season of strong to severe depressions. Ancient acupuncture books say depression means conjested liver with low activity and suggest to stimulate with acupuncture the liver 
meridian. Since the liver is maximally active during the spring, if the liver is congested and cannot become active enough in the spring, the person feels depressed.

The ancient acupuncture books recommend: since spring is the season of depression, during the spring "the people should be encouraged and rewarded, but not punished". 5

\section{The heart is most active in the summer}

Statistical data show that most of the people, who suffer of heart disease, die in the summer. ${ }^{5}$ This could only be explained by maximal activity of the heart during the summer.

\section{The spleen is most active in the late summer}

Both, Spleen and Liver, are blood-related organs. In the daily energy cycle, the liver is active first then the Spleen. In the yearly energy cycle, the liver is active in the spring and the spleen is active in the late summer.

The lungs are most active in the fall: Statistical data show that the number of people dying of lung diseases is maximal in the fall. ${ }^{5}$ Only maximal activity of the lungs during the fall could explain this and the ancient acupuncture books ${ }^{1}$ say exactly this.

\section{The kidneys are most active during the winter}

Statistical data show that the deaths from kidney's diseases have maximum in the winter. This indicates that the kidneys are most active during the winter. Also, contemporary study of Dr. Halberg found from the statistical data of the Department of Health in Minnesota that the number of people dying of sclerosis was maximal in January.,10 This should not be surprising because the winter is a period of low metabolic rate and slow blood movement, which would aggravate sclerosis.

\section{Mother-son law}

The Law of Five Elements, which reflects the daily energy cycle of organs' activation, is also depicted in Figure 4. At 11a.m., the heart is maximally active, at 1p.m., the small intestines are maximally active, at 3p.m. the lungs are most active, at 5p.m. - the large intestines, at 7p.m. - the kidneys, at 9p.m. - the bladder, etc. This is the daily cycle of organ activity.

The yearly cycle of organ activity has the same sequence. In both cycles the organ with earlier activity is considered the mother, the organ that become active after it is called the son. It is known as Mother-Son Law and it is an important law, which every practicing acupuncturist needs to know and consider in his practice.For example:

A. The Liver and Gallbladder (Wood), which are most active in the spring, is the mother of the Heart and Small Intestines (Fire), which are most active in the summer. The Liver and Gallbladder (Wood), which are most active in the spring, is the son of the Kidney and Bladder (Water), which is most active in the winter (Figure 4) (Figure 5).

B. The Heart and Small Intestines (Fire), which are most active in the summer, is the mother of the Spleen and Stomach (Earth), which are most active in the Late Summer. The Heart and Small Intestines (Fire), which are most active in the summer, is the son of the Liver and Gallbladder (Wood), which are most active in the Spring (Figure 4) (Figure 5).
C. The Lungs and Large Intestines (Metal), which are most active in the fall, are the mother of the Kidneys and Bladder (Water), which are most active in the winter. The Lungs and Large Intestines (Metal), which are most active in the fall, are the son of the Spleen and Stomach (Earth), which are most active in Late Summer (Figure 4) (Figure 5).

D. The Kidney and Bladder (Water), which are most active in the winter, is the mother of the Liver and Gall Bladder (Wood), which is most active in the spring. The Kidney and Bladder (Water), which are most active in the winter, is the son of the Lungs and Large Intestines (Metal), which are most active in the Fall (Figure 4) (Figure 5).

The flux of energy (Chi) to the son depends on the mother: when the mother is stimulated, the son is automatically stimulated. This applies to clinical practice in the following way: If a meridian is empty, stimulate its mother. If it is full, disperse the son. ${ }^{1}$ For example, if the energy of the Kidneys (winter) is weak and the Kidney meridian empty, stimulate the mother meridian of the Kidneys, which is Lungs (fall). If the Lungs are weak and the Lungs' meridian empty, stimulate their mother meridian, which is Spleen (Late Summer), etc. If the meridian of the Kidneys (winter) is full, disperse the son - the Liver (spring). If the meridian of the Lungs (fall) is full, disperse the son the Kidneys (winter), etc.

Acupuncture treatment of each mother point always supplements energy, while acupuncture treatment of each son's point always drains energy. Isn't it so in our daily life? The mothers are always supplementing and the sons are always draining and it seems it has been this way always - in ancient time and today.

The Mother-son Law is used when sensitive patients are treated. If the pulse of a sensitive patient is quick with many beats per minute, the heart meridian must not be touched because the patient will collapse. Instead, the gallbladder meridian must be sedated, which will calm down the heart. The ancient acupuncture sources say:

A. "When people are injured by severe cold during the winter, sickness will recur in the spring.

B. When people are hurt by wind in the spring, they will not be able to retain their food in the summer.

C. When people are hurt by extreme heat in the summer, they will get intermittent fever in the fall.

D. When people are hurt by humidity in the fall, they will get a cough in the winter."

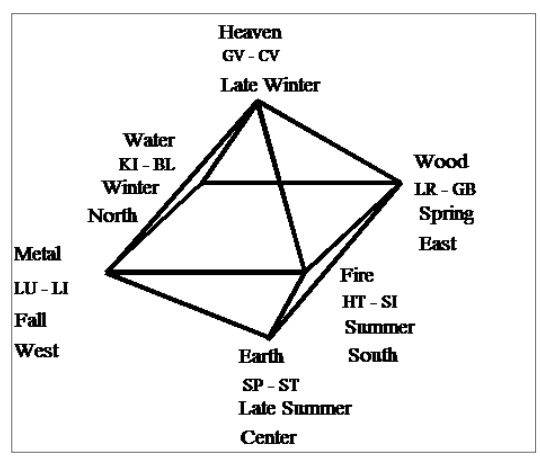

Figure $\mathbf{5}$ The yearly cycle of organ activity has the same sequence. 


\section{Husband-Wife Law}

It is known from contemporary studies ${ }^{12}$ that for right-handed men the processes in their left hemisphere of the brain run faster than those in the right hemisphere. Even the hair grows faster on the left half of their head. Oxidation is the most basic process in the brain. According to the Krebs' cycle, it is a transfer of electrons, while protons are emitted as side products. More active left hemisphere means more active oxidation. As a result on the left half of the head more positive electric charges will be emitted as side products, than on the right. This is exactly what we measured. The left half of the head of right-handed males was electrically more positive than the right (the measurements were done during the day).

Since the processes in the left hemisphere of the brain of righthanded men run faster, and the left hemisphere rules the right arm and leg, one can expect the processes in the men's right arm and leg to run faster. Indeed, contemporary measurements of arm temperature showed that the right arm of right-handed men has a higher temperature than the left, which means that the processes there run faster during the day. ${ }^{5}$

Therefore, the left-right distribution of electrical charges on the surface of the male body is ruled and determined by the different speed of processes in the brain hemispheres, which rule the opposite halves of the body. The dynamic alternative switch in the activity of both hemispheres rules and determines the observed dynamic switch of the left-right electrical asymmetry.

The active left brain hemisphere of right-handed men rules not only the right leg and arm, but also the left half of the body. Thus, more positive charges can be expected on the left half of the back. Indeed, we measured in 1974 electric difference left - right $^{14}$ and T. Moss and K. Johnson reported in 1975 that they also measured such difference..$^{13}$ It was called 'semiconducting effect', because when points equidistant from the backbone are connected to a sensitive electrometer, a current is detected running in one direction.

It was also found that in cases of pathology the difference left right is always 3 to 4 times larger than the normal, which can be used for diagnostic purposes. Y. Manaka et al. in their book ${ }^{2}$ on p. 33 claim that such pathology is usually caused by bias in behavior, such as swimming mainly with one hand, playing tennis with one hand, even chewing on one side only, etc. This natural small difference left - right has been known for thousands of years, and since it is weak, it was called "husband-wife law". ${ }^{1}$ Here is the proof that the relations: right arm - left half of the torso, left arm - right half of the torso were known since ancient time. The ancient acupuncture book Huang Ti Nei Ching Sue Wen ${ }^{1}$ says:

"Those who are expert in using the needles for acupuncture... use the right hand to treat the illness of the left side, and they use the left hand to treat the illness of the right side". ${ }^{4}$

\section{Gender differences in the left-right asymmetry}

According to the ancient acupuncture book, ${ }^{1}$ the men are Yang, which means 'active'. Contemporary studies indicate that the temperature of the male body is usually higher, which means that the processes in the male body are more active. The ancient books say the women are Yin, which means 'passive'. Contemporary studies indicate that the temperature of the female body is usually lower, which means that the processes in her body are more passive. This explains why each time the wife tries to set the thermostat in the house to higher temperature, she finds it lowered by the husband.
The male and female bodies are wired differently. "Man's left hand and foot are not so strong as are his right hand and foot". For women the opposite is true - woman's right hand and foot are not so strong as her left hand and foot.

"Man's hearing and eyesight are not so clear on his right side as they are on his left side". For women the opposite is true - woman's hearing and eyesight are not so clear on her left side as they are on her right side.

The 'semiconducting effect' reported by T. Moss and K. Johnson in $1975^{13}$ was a current running between points equidistant from the backbone. For men this current was always running in the direction right - left, for women in the direction left - right during the day.

Concerning the left-right asymmetry, men's and women's hands and arms, legs and feet are opposite in activity and electric charges as in a snap-on button, so that male and female can complement each other. If males and females were such antipodes, they would need different approaches when diagnosed and treated.

\section{Diagnosis}

Indeed, in ancient time "the procedure of palpitation (of the pulse) differed according to the sex of the patient. If it was a woman, her right pulses had to be taken first; if it was a man, his left pulses had to be examined first." because "woman's right pulse indicates disorder, while her left pulse indicates order; man's left pulse indicates disorder, while his right pulse indicates order."

Thus, for diseases in men palpitate the pulse on their left arm, which is weaker, to detect earlier and easier the pulse disorder indicating oncoming disease. For diseases in women palpitate the pulse on their right arm, which is weaker, to detect earlier and easier the pulse disorder indicating oncoming disease. However, the pulses of both hands were checked for each gender to monitor the balance left - right.

\section{Healing}

In ancient time: "Those who were experts in using the needles for acupuncture followed the female principle Yin to draw out Yang, and they followed the male principle Yang to draw out Yin. They used the right hand to treat illness of the left side, and the left hand to treat illness of the right side. ${ }^{1}$

Nowadays, usually both hands are treated with acupuncture for cure in both genders. However, considering the left-right asymmetry in both genders, since the right hand in men and the left hand in women are hotter and have stronger biocurrents, the stimulating effect of acupuncture can be expected to be stronger when done on the right hand in men and the left hand in women.

Are the left-right differences permanently fixed or do they change? During the night they change to opposite. However, this will not be discussed here since it does not relate directly to acupuncture practice, which is usually done only during the day. Additional information can be found in the author's book: Maria Kuman, Eastern Wisdom and Modern Science about Health \& Happiness. ${ }^{15}$

\section{Conclusion}

The circulations of energy along the chain of organs during the day and during the year were discussed and information from the ancient acupuncture books was compared with contemporary studies-their agreement is spectacular. The functional dependence of the organs 
(and their corresponding meridians) was also discussed: in ancient books the strong dependence was called mother-son law, while the weak dependence was called husband-wife law. In this article, we discussed only the proportional dependencies among the organs in the law of five elements. The reciprocal dependences will be subject of another article.

\section{Acknowledgments}

None

\section{Conflict of interest}

Author declares there is no conflict of interest towards the manuscript.

\section{References}

1. Veith I Ed. Huang Ti Nei Ching Sue Wen. Berkley-Los Angeles-London: University of California Press; 1972

2. Manaka Y, Kazuko I, Birch S. Chasing the Dragon's Tail. Paradigm. 1995 p. 453.

3. Kuman M. Modern Aspects of Ancient Acupuncture. 2nd ed. USA: Health and Happiness Books; 1997.

4. Kuman M. Science Speaks to God. 2nd ed.USA: Health and Happiness Books; 2005.
5. Marjorie C, Meehan. Biological Rhythms in Psychiatry and Medicine. JAMA.1970;215(4):645.

6. Halberg F, Peterson RE, Silber RH. Endocrinology. 1956;64(2):222-230.

7. Feigin RD, Kleiner AS, Beisel. Factors affecting circadian periodicity of blood amino acids in man. Metabolism. 1968;17(9):764-765.

8. Spoor RP, Jackson DB. Circadian rhythms: variation in sensitivity of isolated rat artria to acetylcholine. Science. 1966;154(3750):782.

9. Klein KE, Wegmann HM, Brüner H. Circadian rhythm in indices of human performance, physical fitness and stress resistance. Aerosp Med. 1968;39(5):51-518.

10. Halberg F, Haus E, Stephens A. Federation Proceedings. 1959;18:63.

11. Kuman M. Yoga - Health Benefits, Wisdom, and Science. 2nd ed. 1999.

12. Restak R. The Brain. USA: Bantam Books; 1984.

13. Moss T, Johnson K. The Galaxies of Life. In: Kripner \& Rubin, editors. 1975.

14. Moss T, Johnson K. US Conference on Acupuncture. Bethesda, Maryland; 1973.

15. Kuman M. Eastern Wisdom and Modern Science about Health and Happiness. 2nd ed. USA: Health and Happiness Books; 1998. 